\title{
SIMULACIÓN EXPERIMENTAL Y NUMÉRICA DE UN PROCESO DE TREFILADO HÚMEDO DE UN ALAMBRE DE ACERO AL CARBONO
}

\section{EXPERIMENTAL AND NUMERICAL SIMULATION OF THE DAMP WIRE DRAWING PROCESS OF A CARBON STEEL}

\author{
Ennio L. Rojas ${ }^{1} \quad$ Diego J. Celentano ${ }^{2} \quad$ Alfredo A. Artigas $^{3} \quad$ Alberto G. Monsalve $^{3}$ \\ Recibido el 21 de marzo de 2006, aceptado el 5 de noviembre de 2007 \\ Received: March 21, $2006 \quad$ Accepted: November 15, 2007
}

\begin{abstract}
RESUMEN
En este trabajo se presenta un análisis experimental y numérico para la descripción del comportamiento mecánico de un alambre de acero al carbono durante un proceso industrial de trefilado húmedo. En primer lugar, se hizo una campaña experimental de mediciones de fuerza de trefilado de las doce reducciones presentes en el proceso. En segundo lugar, se llevaron a cabo ensayos de tracción con objeto de caracterizar el comportamiento mecánico del material en cada etapa de reducción. En estos ensayos se obtuvieron curvas de tensión-deformación que, en conjunto a las respuestas calculadas a partir de la simulación de los ensayos, permitieron derivar la evolución de los parámetros elásticos y plásticos característicos del material a medida que se reduce su diámetro. De dicha evolución se constató el gran endurecimiento que experimenta el material durante el proceso, el que, a su vez, condiciona fuertemente su trefilabilidad. Las simulaciones se realizaron por medio de un modelo elastoplástico de grandes deformaciones implementado en un programa de cálculo preexistente, basado en el método de elementos finitos, denominado VULCAN. Luego, los parámetros del material obtenidos en los ensayos de tracción fueron considerados en la simulación del proceso de deformación que ocurre durante el paso del alambre a través de los dados. Los resultados de la simulación se consideran aceptables y representativos del comportamiento del alambre en el proceso.
\end{abstract}

Palabras clave: Trefilado de alambres, caracterización experimental, modelación elastoplástica, simulación numérica.

\begin{abstract}
This paper presents an experimental and numerical analysis for the description of the mechanical behaviour of a carbon steel wire during an industrial process of damp drawing. Firstly, an experimental procedure aimed at measuring wire drawing forces in the twelve reductions present in the process was performed. Secondly, tensile tests were carried out in order to characterize the mechanical behavior of the material for each reduction step. The resulting stress-strain curves together with the computed responses obtained from the simulation of such tests allowed to derive the evolution of the representative elastic and plastic material parameters. This parameter evolution clearly reflected the relevant amount of hardening experienced by the material which, in turn, imposes restrictions over the drawing operation. The numerical simulations were conducted via a large strain elasto-plasticity model implemented in an existing finite element-based code called VULCAN. The material parameters obtained in the tensile tests were considered as data for the modeling of the entire wire drawing sequences. The computed results are considered as reasonable and representative of the material response during the deforming process.
\end{abstract}

Keywords: Wire drawing, experimental characterization, elasto-plastic modeling, numerical simulation.

\section{INTRODUCCIÓN}

El proceso de trefilado de un alambre de sección circular consiste en reducir de forma simétrica la sección del mismo al hacerlo pasar, mediante una fuerza de tiro, a través de una hilera o serie de hileras (también denominadas matrices o dados [1]).

\footnotetext{
1 Departamento de Ingeniería Mecánica. Universidad de La Serena. Benavente 980. La Serena, Chile.

2 Departamento de Ingeniería Mecánica y Metalúrgica. Pontificia Universidad Católica de Chile. Av. V. Mackenna 4860. Santiago, Chile. E-mail: dcelentano@ing.puc.cl

3 Departamento de Ingeniería Metalúrgica. Universidad de Santiago de Chile. Av. Bdo. O’Higgins 3363. Santiago, Chile. E-mail: amonsalv@usach.cl
} 
El trefilado húmedo por resbalamiento está formado por un juego de tambores de distintos diámetros dispuestos en dos ejes paralelos que giran a diferentes velocidades. En medio de ellos están ubicados los distintos dados de la serie de trefilación; ver figura 1 . Todo el conjunto está sumergido en un baño de jabón de trefilación.

La particularidad de este proceso es que la reducción de área experimentada por el alambre se encuentra limitada por la relación de velocidades existente entre sus ejes giratorios. Este hecho hace que sólo exista un cierto porcentaje de resbalamiento admisible entre el alambre y los tambores de tiro que pueda compensar el desgaste de los dados; ver figura 2.

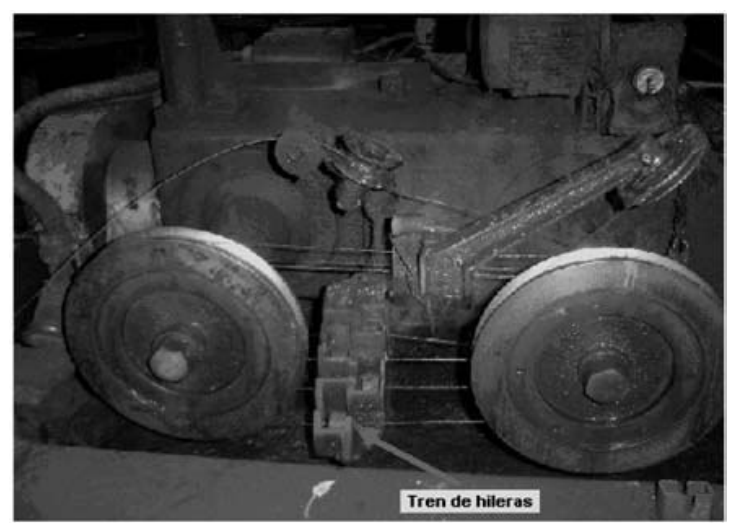

Figura 1. Ubicación del tren de hileras en los tambores de la máquina.

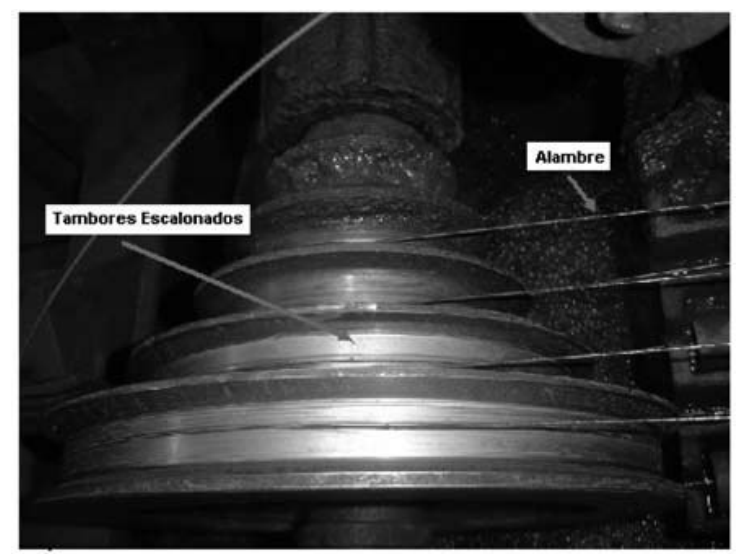

Figura 2. Detalle de los tambores escalonados de la máquina trefiladora.

Uno de los problemas del proceso en condiciones de operación industrial es el corte del alambre cuando se trefilan diámetros pequeños, especialmente en los diámetros nominales de $0,8 \mathrm{~mm}$ y $0,9 \mathrm{~mm}$, lo que implica pérdida de tiempo y paradas del proceso continuo de trefilación.
Cabe señalar que el material de entrada ya cuenta con un gran endurecimiento previo al proceso debido a que el alambre experimenta una deformación cercana al $90 \%$ de deformación producto de la trefilación en seco anterior. En las últimas hileras de este proceso la velocidad de operación alcanza los $10 \mathrm{~m} / \mathrm{s}$.

Para lograr predecir la evolución de las propiedades mecánicas del alambre durante el proceso de deformación, se presentan en este trabajo los resultados de una campaña experimental que contempló ensayos de trefilación a escala de laboratorio para los distintos dados correspondientes al proceso de trefilado húmedo. En dichos ensayos se midieron las cargas de trefilación en cada pasada.

Además, el alambre de cada reducción fue sometido al ensayo de tracción convencional con el fin de derivar, a partir de la curva de tensión ingenieril v/s deformación ingenieril, la evolución de los parámetros elásticos y plásticos característicos del material a medida que se reduce su diámetro.

Luego, se simuló el comportamiento del material en cada uno de los ensayos de tracción a través de un modelo elastoplástico de grandes deformaciones con endurecimiento potencial (expresado por la ecuación de Hollomon) implementado en un programa de cálculo preexistente denominado VULCAN [2].

Los parámetros obtenidos en la simulación numérica del ensayo de tracción se consideraron en la simulación del proceso de trefilación, utilizando el mismo modelo antes mencionado, para cada uno de los dados. De la comparación de los resultados numéricos con los experimentales se verificó que el modelo entrega una respuesta mecánica representativa de la experimentada por el material en el proceso industrial.

\section{TRABAJO EXPERIMENTAL}

\section{Material}

El material caracterizado fue el correspondiente a un alambre del tipo ER70S-6 de la AWS (American Welding Society). La composición química media del material se presenta en la tabla 1. Dicho alambre fue obtenido a la salida del trefilado seco existente en la empresa Indura Solcon. En la tabla 2 se entrega un detalle de los diámetros de reducción para cada etapa del proceso de trefilado húmedo para dos medidas de diámetros finales en la última reducción. 
Tabla 1. Composición química media del alambre.

\begin{tabular}{|c|c|c|c|c|c|c|c|c|}
\hline $\mathbf{C}$ & $\mathbf{S i}$ & $\mathbf{M n}$ & $\mathbf{P}$ & $\mathbf{S}$ & $\mathbf{C r}$ & $\mathbf{M o}$ & $\mathbf{N i}$ & $\mathbf{C u}$ \\
\hline 0,064 & 0,968 & 1,45 & 0,011 & 0,014 & 0,034 & 0,016 & 0,022 & 0,026 \\
\hline
\end{tabular}

Tabla 2. Diámetros de reducción del proceso de trefilado húmedo.

\begin{tabular}{|c|c|c|}
\hline Reducción & $\begin{array}{c}\text { Diámetro } \\
0,9(\mathrm{~mm})\end{array}$ & $\begin{array}{c}\text { Diámetro } \\
0,8(\mathrm{~mm})\end{array}$ \\
\hline diámetro inicial & 2,02 & 1,75 \\
\hline 1 & 1,89 & 1,60 \\
\hline 2 & 1,75 & 1,47 \\
\hline 3 & 1,60 & 1,35 \\
\hline 4 & 1,47 & 1,24 \\
\hline 5 & 1,35 & 1,13 \\
\hline 6 & 1,24 & 1,05 \\
\hline 7 & 1,13 & 0,97 \\
\hline 8 & 1,05 & 0,90 \\
\hline 9 & 0,97 & 0,84 \\
\hline 10 & 0,90 & 0,77 \\
\hline
\end{tabular}

\section{Ensayos de tracción}

La configuración geométrica utilizada para el ensayo de tracción corresponde a la recomendada por la norma internacional ASTM E8 [3]. La velocidad establecida para las pruebas fue de $5 \mathrm{~mm} / \mathrm{min}$ considerando una distancia extensométrica de $50 \mathrm{~mm}$. Además, se utilizaron mordazas especiales para evitar el corte del alambre producido por el efecto de cizallamiento en el alambre que producen las mordazas planas convencionales. El detalle de la ubicación y montaje del alambre en las mordazas se muestra en la figura 3 (las mordazas utilizadas en este trabajo fueron proporcionadas por la empresa Indura Solcon).

En la figura 4 se grafican las curvas tensión-deformación medidas para los diferentes diámetros de alambre $\left(\mathrm{d}_{0} \mathrm{y}\right.$ $\mathrm{d}_{12}$ corresponden a $2,02 \mathrm{~mm}$ y $0,77 \mathrm{~mm}$, respectivamente; ver tabla 2). En dichas curvas se puede apreciar el comportamiento que presenta el material a medida que avanza su deformación: aumenta el endurecimiento relativo en función de la reducción de área, se mantiene constante el módulo de Young (el que corresponde a $210 \mathrm{GPa}$ ) y, además, se reduce de manera paulatina el porcentaje de elongación a rotura a lo largo del proceso acompañado de una disminución de la zona de estricción.

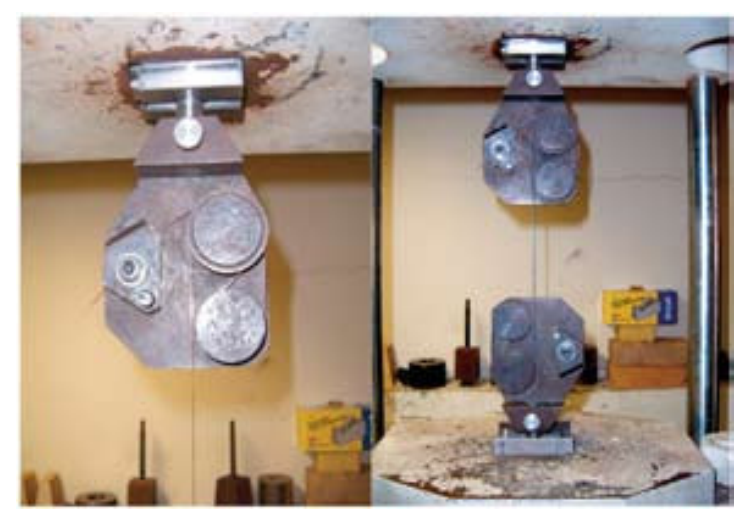

Figura 3. Detalle de la ubicación y montaje del alambre en las mordazas.

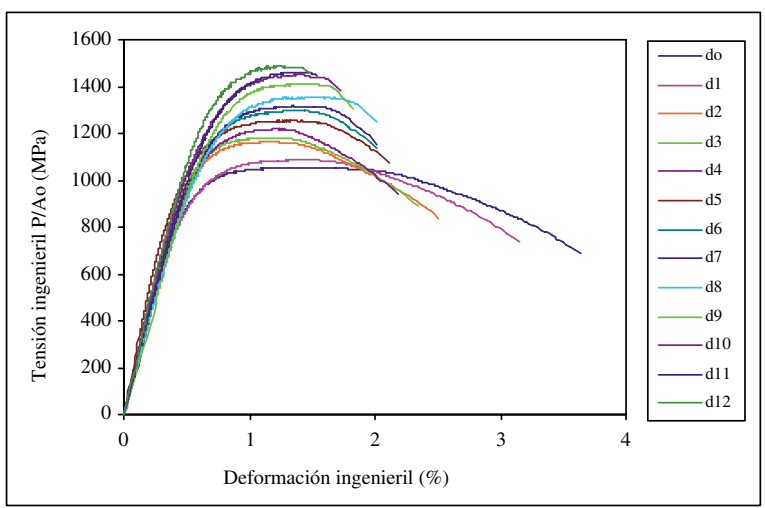

Figura 4. Evolución de la curva de tensión ingenieril v/s deformación ingenieril.

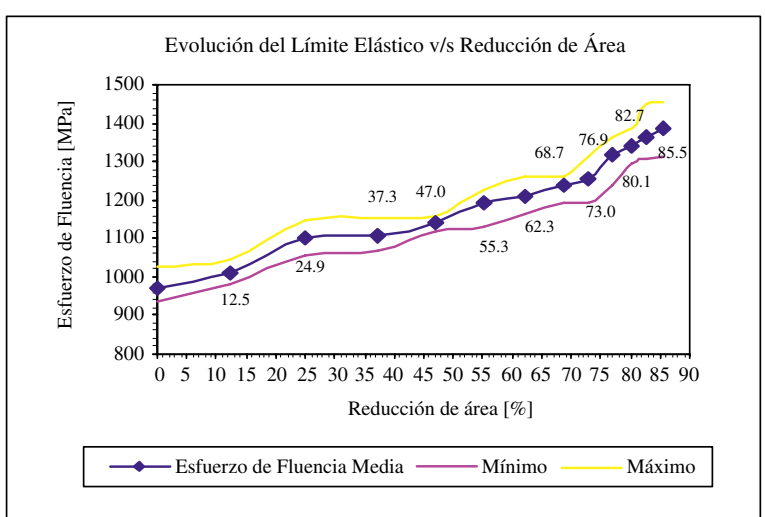

Figura 5. Evolución del límite elástico a través de los dados de reducción como \% de reducción de área.

En la figura 5 se muestra cómo el límite elástico del material aumenta en función del \% de reducción acumulado en el proceso. Cabe señalar que el esfuerzo último presenta una tendencia similar, lo que indica que el material desarrolla un endurecimiento apreciable a medida que aumenta la deformación por trefilación. 
Es un hecho conocido que la velocidad de deformación no afecta mayormente al endurecimiento de los aceros en temperaturas cercanas a la del ambiente [4-6]. Los resultados de la figura 6 ratifican este hecho, ya que en la misma se observa que las curvas de tensión-deformación del alambre de diámetro $\mathrm{d}_{12}$ obtenido a partir del proceso de trefilado para dos velocidades diferentes de trefilación $(0,0013 \mathrm{~m} / \mathrm{s}$ y $10 \mathrm{~m} / \mathrm{s}$ para condiciones de laboratorio y planta industrial, respectivamente) prácticamente coinciden.

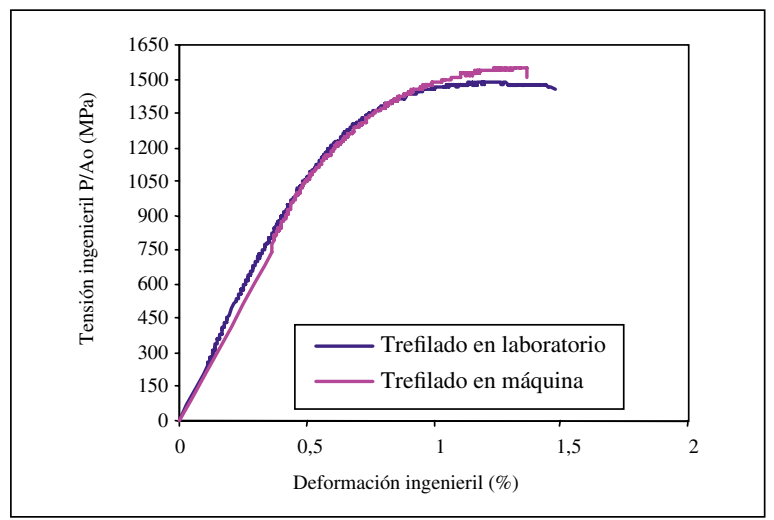

Figura 6. Curva de tensión ingenieril v/s deformación ingenieril comparativa para diámetro de salida trefilado a distintas velocidades.

Estos resultados concuerdan con los resultados experimentales reportados en [4-6], donde se muestra que en los materiales con estructura BCC la influencia de la velocidad de deformación en el ensayo de tracción decrece con la deformación a la que el material es sometido. En el presente estudio debe mencionarse que el alambre de entrada al proceso trefilado húmedo ya fue sometido a una trefilación en seco cercana al $90 \%$, para luego sufrir durante el trefilado húmedo una nueva deformación plástica cercana al $80 \%$.

\section{Trefilación}

La trefilación del alambre se realizó en laboratorio a una velocidad de trefilación de $80 \mathrm{~mm} / \mathrm{min}(0,0013 \mathrm{~m} / \mathrm{s})$ en una máquina de tracción universal, tal como se muestra en la figura 7. En el ensayo se utilizaron las mismas hileras que se emplean en el proceso de trefilado húmedo industrial (los ángulos de las hileras corresponden a $6^{\circ}$ para las primeras hileras del tren y a $5^{\circ}$ para las finales de acabado). En la tabla 3 se entrega un detalle de los valores máximos y mínimos de las fuerzas de trefilación obtenidas. Puede notarse que las fuerzas medidas presentan una baja dispersión y que las mismas se reducen a lo largo del proceso de reducción.

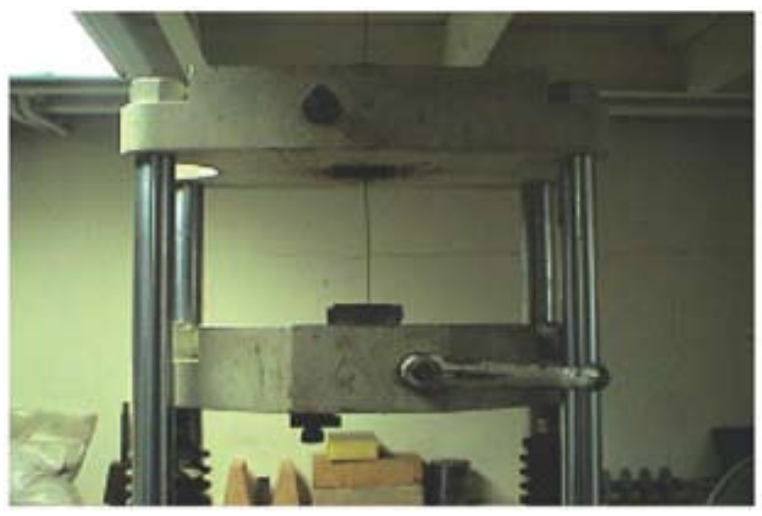

Figura 7. Ensayo de trefilado en una máquina de tracción.

Tabla 3. Resultados del ensayo de trefilación para cada dado.

\begin{tabular}{|c|c|c|c|}
\hline Hilera & $\begin{array}{c}\text { Fuerza de } \\
\text { trefilado }(\mathrm{KN})\end{array}$ & Mínimo & Máximo \\
\hline 1,89 & 1,20 & 1,17 & 1,23 \\
\hline 1,75 & 1,23 & 1,22 & 1,25 \\
\hline 1,6 & 1,10 & 1,08 & 1,12 \\
\hline 1,47 & 0,88 & 0,84 & 0,90 \\
\hline 1,35 & 0,83 & 0,80 & 0,84 \\
\hline 1,24 & 0,70 & 0,67 & 0,75 \\
\hline 1,13 & 0,62 & 0,60 & 0,63 \\
\hline 1,05 & 0,53 & 0,51 & 0,55 \\
\hline 0,97 & 0,50 & 0,48 & 0,51 \\
\hline 0,90 & 0,41 & 0,40 & 0,43 \\
\hline 0,84 & 0,33 & 0,30 & 0,36 \\
\hline 0,77 & 0,35 & 0,33 & 0,36 \\
\hline
\end{tabular}

\section{SIMULACIÓN NUMÉRICA}

\section{Ensayos de tracción}

Con los resultados experimentales del ensayo de tracción se procedió a realizar una simulación de dicho ensayo con el objetivo de obtener los parámetros de endurecimiento $A^{p}$ y $n^{p}$ de la ley potencial de Hollomon [1] expresada de la siguiente forma:

$$
C^{p}=A^{P}\left(e_{0}^{p}+e^{p}\right)^{n^{p}}
$$

donde $C^{p}$ es la función de endurecimiento y $\bar{e}_{0}^{p}$ es una medida de deformación tal que $C_{0}=\left(\bar{e}_{0}^{p}\right)^{n^{p}}$, donde $\mathrm{C}_{0}$ 
es el límite elástico inicial del material. A partir de la metodología reportada en [7], se obtuvieron el par de valores que mejor ajustaron a las curvas experimentales de tensión-deformación mostradas en la figura 4. Los correspondientes valores se presentan en la tabla 4 .

Tabla 4. Parámetros de endurecimiento $A^{p}$ y $n^{p}$ para cada diámetro del alambre.

\begin{tabular}{|c|c|c|c|}
\hline Diámetro & $\mathrm{A}^{\mathrm{p}}$ & $\mathrm{n}^{\mathrm{p}}$ & \% reducción \\
\hline 2,02 & 1180 & 0,0200 & 0 \\
\hline 1,89 & 1190 & 0,0177 & 12,46 \\
\hline 1,75 & 1250 & 0,0132 & 24,95 \\
\hline 1,60 & 1275 & 0,0122 & 37,26 \\
\hline 1,47 & 1330 & 0,0128 & 47,04 \\
\hline 1,35 & 1365 & 0,0133 & 55,34 \\
\hline 1,24 & 1410 & 0,0145 & 62,32 \\
\hline 1,13 & 1440 & 0,0125 & 68,71 \\
\hline 1,05 & 1480 & 0,0135 & 72,98 \\
\hline 0,97 & 1520 & 0,0123 & 76,94 \\
\hline 0,90 & 1560 & 0,0123 & 80,15 \\
\hline 0,84 & 1565 & 0,0114 & 82,71 \\
\hline 0,77 & 1600 & 0,0110 & 85,47 \\
\hline
\end{tabular}

En la tabla 4 se aprecia que el parámetro $A^{p}$ presenta un comportamiento creciente ligado al mayor endurecimiento que va experimentando el material a medida que se reduce su diámetro. El parámetro $n^{p}$, por el contrario, no presenta una tendencia clara de variación.

En la figura 8 se presentan los resultados correspondientes a la deformación de rotura de la tensión equivalente de Von Mises $\sigma_{e q}$ y de deformación plástica efectiva $\bar{e}^{p}$ para los diámetros $2,02 \mathrm{~mm}, 1,24 \mathrm{~mm}$ y $0,77 \mathrm{~mm}$, los cuales son representativos de las etapas inicial media y final del proceso. Se puede observar que el valor relativo de la deformación plástica efectiva disminuye a través de las sucesivas reducciones. La tensión equivalente, por el contrario, aumenta en función de la reducción concentrándose de manera gradual en el cuello de la probeta cuya formación va atenuándose para diámetros menores ( $\mathrm{D}$ es el diámetro de rotura de la probeta). Se ve claramente que el endurecimiento relativo del alambre le hace perder sus propiedades de ductilidad a lo largo de las sucesivas reducciones.

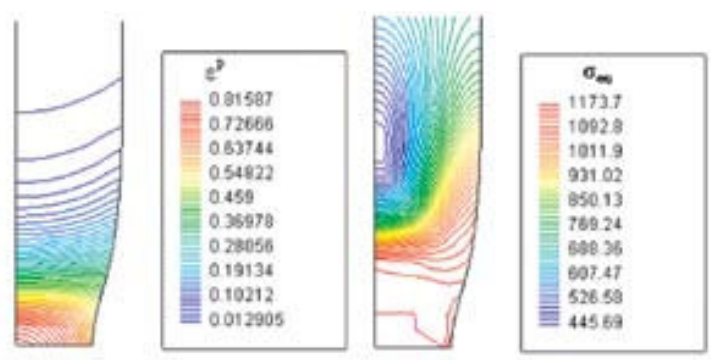

$\mathrm{Do}=2,02 \mathrm{~mm} ; \mathrm{D} / \mathrm{Do}=0,7$
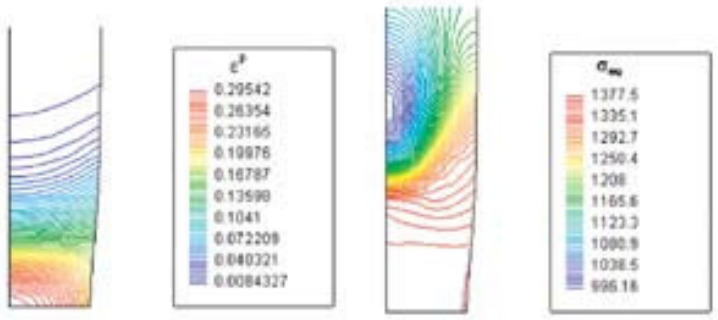

$\mathrm{Do}=1,24 \mathrm{~mm} ; \mathrm{D} / \mathrm{Do}=0,87$
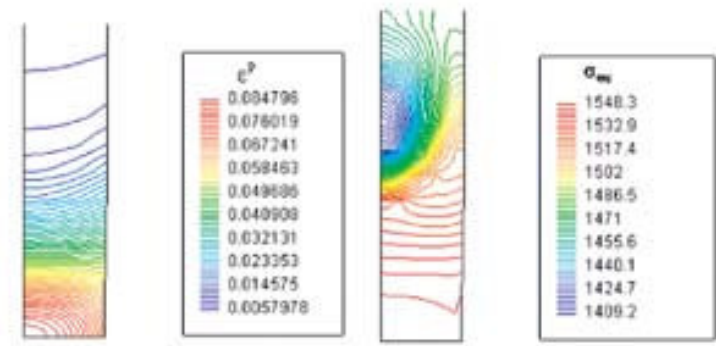

$\mathrm{Do}=0,77 \mathrm{~mm} ; \mathrm{D} / \mathrm{Do}=0,96$

Figura 8. Resultados de deformación plástica efectiva y de tensión equivalente de Von Mises para tres diámetros en el ensayo de tracción.

\section{Trefilación}

Para la simulación del proceso de trefilado se realizaron discretizaciones espaciales (mallas de elementos finitos) para cada diámetro de reducción en conjunto con las distintas configuraciones geométricas de las hileras de trefilado. El modelo numérico utiliza los parámetros de endurecimiento $A^{p}$ y $n^{p}$ de la ley de Hollomon obtenidos de la simulación del ensayo de tracción. Para la simulación de este proceso se sigue la metodología reportada en [6].

En la figura 9 se presentan los contornos estacionarios de deformación plástica efectiva relativa y de tensión equivalente de Von Mises. Se aprecia que la deformación alcanza sus valores máximos en la zona recta del dado, que es el lugar en donde se genera el diámetro de salida del alambre. Por otro lado, en la tensión equivalente se observa un aumento en función de la etapa de reducción, 
lo que se explica por el gran endurecimiento que adquiere el material a lo largo del proceso.

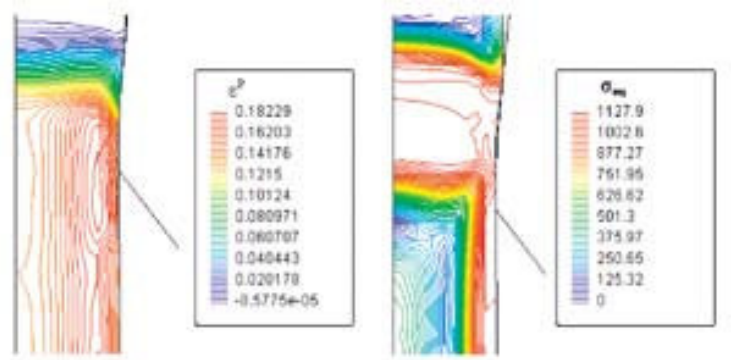

$\mathrm{Do}=2,02 \mathrm{Df}=1,89 \mathrm{Df} / \mathrm{Do}=0,93$

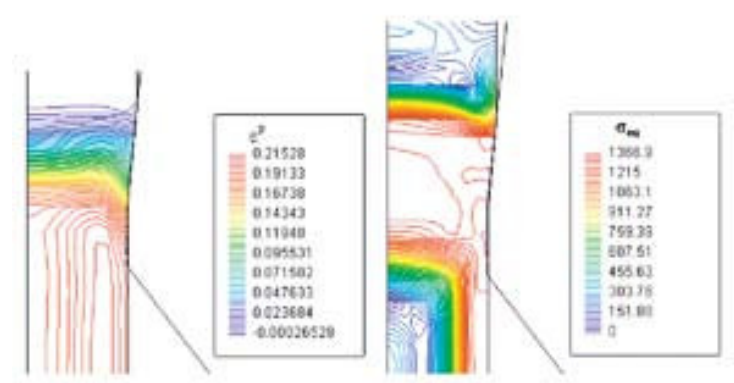

$\mathrm{Do}=1,24 \mathrm{Df}=1,13 \mathrm{Df} / \mathrm{Do}=0,91$

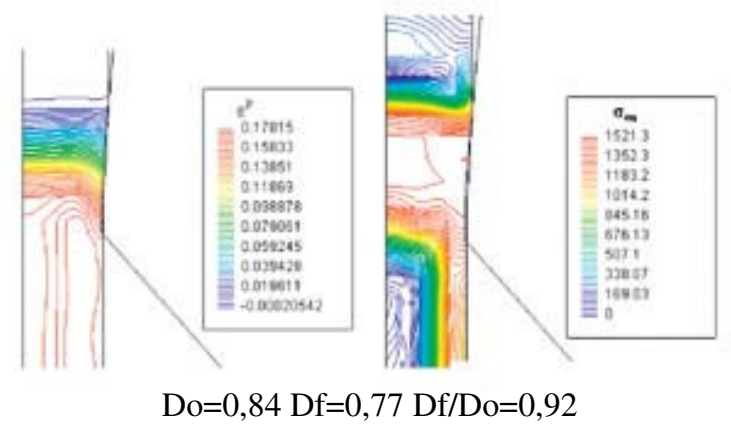

Figura 9. Resultados de deformación plástica efectiva y de tensión equivalente de Von Mises para tres diámetros en el ensayo de trefilación.

Los resultados para las cargas de trefilación se presentan en la figura 10, donde se aprecia que la simulación predice de manera satisfactoria las mediciones realizadas en laboratorio.

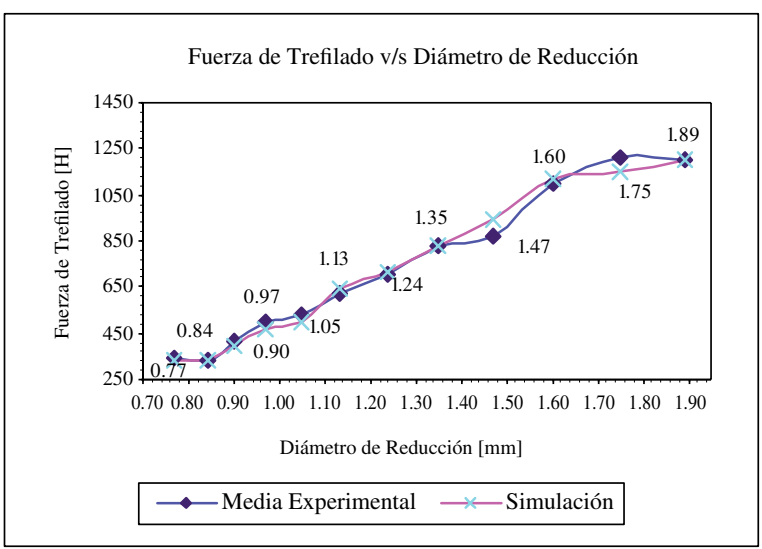

Figura 10. Fuerza de trefilado v/s diámetro de reducción.

\section{CONCLUSIONES}

Se ha presentado en este trabajo un análisis experimental y numérico del comportamiento mecánico de un alambre de acero al carbono durante un proceso industrial de trefilado húmedo. Se ha observado que el material no sólo experimenta un gran endurecimiento a medida que se va deformando al pasar por las sucesivas hileras del tren de trefilado sino que, además, va perdiendo ductilidad durante este proceso.

El análisis experimental y numérico del ensayo de tracción llevado a cabo para los diferentes diámetros de alambre ha permitido caracterizar de manera adecuada el comportamiento mecánico del material para cada una de las etapas del proceso de trefilado.

Por otro lado, las cargas de trefilado calculadas a través de la simulación numérica de la secuencia completa de reducción han presentado valores muy cercanos a los medidos en laboratorio. Este aspecto es operativamente muy importante, ya que puede permitir en futuros desarrollos relacionar niveles de esfuerzos de trefilado con diversos fenómenos que se producen en los procesos de trefilado industriales, tales como cortes de alambre y desgastes prematuros de los dados.

\section{AGRADECIMIENTOS}

Los autores desean expresar su agradecimiento a la empresa Indura Solcon por su colaboración en este trabajo y el soporte brindado por CONICYT (Proyecto Fondecyt 1060139). 


\section{REFERENCIAS}

[1] M. Groover. "Fundamentals of modern manufacture". Lehigh University, Prentice Hall. 1997.

[2] E. Cabezas and D. Celentano. "Experimental and numerical analysis of the tensile test using sheet specimens". Finite Elem. Anal. Des. Vol. 40, pp. 555-575. 2004.

[3] Annual Book of ASTM Standards. Section 3: Metals Test Methods and Analytical Procedures. 1988.

[4] S. He, P. Van Houtte, A. Van Bael, F. Mei, A. Sarban, P. Boesman, F. Gálvez and J. Atienza. "Strain rate effect in high-speed wire drawing process". Modelling and Simulation in Materials Science and Engineering, 10, pp. 267-276. 2002.
[5] F. Gálvez, J.M. Atienza, J. Ruiz y M. Elices. "Influencia de la velocidad de deformación en el comportamiento mecánico de alambres de acero trefilados". Anales de mecánica de la fractura. Vol. 18, pp. 185-189. Bayona, España. 2001.

[6] F. Gálvez, J.M. Atienza, J. Ruiz y M. Elices. "El efecto de la velocidad de deformación en la rotura de alambres de acero durante el trefilado". Anales de mecánica de la fractura. Vol. 19, pp. 73-78. Gerona, España. 2002.

[7] D. Celentano, S. Villalobos, A. Artigas and A. Monsalve. "Thermomechanical simulation and experimental validation of the wire drawing process". Computational methods for Coupled Problems in Sciences and Engineering, M. Papadrakakis, E. Oñate and B. Schrefler (Eds.), CIMNE. 2005. 